\title{
Close pairs of galaxies with different activity levels
}

\section{T. A. Nazaryan ${ }^{1}$, A. R. Petrosian ${ }^{1}$, A. A. Hakobyan ${ }^{1}$, B. J. McLean ${ }^{2}$, and D. Kunth ${ }^{3}$}

\author{
${ }^{1}$ Byurakan Astrophysical Observatory, 0213 Byurakan, Aragatsotn Province, Armenia \\ email: nazaryan@bao.sci.am \\ ${ }^{2}$ Space Telescope Science Institute, 3700 San Martin Drive, Baltimore, MD 21218, USA \\ ${ }^{3}$ Institut d'Astrophysique de Paris, 98 bis Bd Arago, 75014 Paris, France
}

\begin{abstract}
We selected and studied 180 pairs with $\mathrm{d} V<800 \mathrm{~km} \mathrm{~s}^{-1}$ and $D \mathrm{p}<60 \mathrm{kpc}$ containing Markarian (MRK) galaxies to investigate the dependence of galaxies integral parameters, star-formation (SF) and active galactic nuclei (AGN) properties on kinematics of pairs, their structure and large-scale environments. Projected radial separation $D$ p and perturbation level $P$ are better measures of interaction strength than $\mathrm{d} V$. The latter correlates with the density of large-scale environment and with the morphologies of galaxies. Both galaxies in a pair are of the same nature, the only difference is that MRK galaxies are usually brighter than their neighbors. Specific star formation rates (SSFR) of galaxies in pairs with smaller $D \mathrm{p}$ or $\mathrm{d} V$ is in average 0.5 dex higher than that of galaxies in pairs with larger $D \mathrm{p}$ or $\mathrm{d} V$. Closeness of a neighbor with the same and later morphological type increases the SSFR, while earlier-type neighbors do not increase SSFR. Major interactions/mergers trigger SF and AGN more effectively than minor ones. The fraction of AGNs is higher in more perturbed pairs and pairs with smaller $D$ p. AGNs typically are in stronger interacting systems than star-forming and passive galaxies. There are correlations of both SSFRs and spectral properties of nuclei between pair members.
\end{abstract}

Keywords. galaxies: general, interactions, starburst, active, peculiar

\section{Introduction}

Interactions/mergers of galaxies are considered as important processes influencing morphological, stellar and chemical evolution of galaxies. Interactions/mergers can enhance star formation rates (SFRs) in galaxies as is revealed by numerous observations of close pairs (e.g. Larson \& Tinsley 1978, Lambas et al. 2003, Li et al. 2008, Ellison et al. 2008, 2013, Hwang et al. 2010 ). The main physical processes responsible are gas inflow toward nuclear regions of galaxies due to global torques and, probably, gas fragmentation into massive and dense clouds and rapid SF therein (e.g. Mihos \& Hernquist 1996, Hopkins et al. 2013). The triggering mechanism of AGN is often considered to be the same as that of the enhanced nuclear SF (e.g. Ellison et al. 2008, 2011, Wild, Heckman \& Charlot 2010, Liu et al. 2012).

Large-scale environments, morphologies of both galaxies and mass ratios can affect on frequency and efficiency of enhanced SF and AGN triggering by interactions and merging (e.g. Sol Alonso et al. 2006, Hwang et al. 2010, Scudder et al. 2012, Li et al. 2008, Ellison et al. 2008, 2010, 2011, Cox et al. 2008, Lambas et al. 2012). The facts, that not all galaxies with high SFR are interacting ones, as well as that not all interacting galaxies have high SFR, support the hypothesis that internal properties of galaxies are also an important factor determining enhanced SF (Lambas et al. 2003, Sol Alonso et al. 2006). 
The aim of this study is to investigate the connections between interaction with a close neighbor and nuclear activity and/or enhanced SF in galaxy pairs using the sample of close neighbors of MRK galaxies from Nazaryan et al. (2012). The complete study is presented in Nazaryan et al. (2014). A similar study of Second Byurakan Survey galaxies is presented in Nazaryan (2014).

\section{Sample}

The starting point to create our sample of pairs is the catalog of MRK galaxies. The original catalog (Markarian et al. 1989) features 1545 bright galaxies mostly having starburst properties and/or AGNs. In Petrosian et al. (2007), homogeneously measured parameters of MRK galaxies are presented. Results of a close neighbors search for MRK galaxies are published in Nazaryan et al. (2012). For the current study, the subsample of 217 pairs of galaxies containing at least one MRK galaxy is selected. For this study, we classified morphologies of MRK galaxies and neighbors by using SDSS color images. We also classified sample pairs in terms of morphological perturbations (see e.g. Liu et al. 2012, Lambas et al. 2012) by 4 levels: $P=0$ : unperturbed pairs, $P=1$ : slightly perturbed, $P=2$ : highly perturbed, $P=3$ : mergers. We described the large-scale environments of each pair by average large-scale density $\Sigma$. For statistics we also included SFRs of galaxies and nuclear emission-line classification (BPT) of their SDSS spectra.

\section{Statistics and discussions}

The dependence of visually detected perturbation level $P$ on $\mathrm{d} V$ and $D$ p is worthy to mention. Disturbance level $P$ correlates only with $D$ p, while $\mathrm{d} V$ and $D$ p do not correlate with each other. The closer pairs are more disturbed. This is the result of different nature of $D \mathrm{p}$ and $\mathrm{d} V$. Pairs with larger $\mathrm{d} V$ correspond to environments with higher densities $\Sigma$. Therefore while $D$ p is a measure of interaction strength, the variation of $\mathrm{d} V$ mainly reflects change of large-scale environments. The SSFRs of barred galaxies do not differ from those of unbarred ones significantly.

We compared properties of neighbors with those of MRK galaxies. Mean absolute $r$ mag of neighbors is fainter by $0.9 \mathrm{mag}$ than that of MRK galaxies. Morphologies of neighbors correlate significantly with those of MRK galaxies and are slightly later. Neighbor galaxies have the same distributions by SSFRs, BPT types and colors, and the same fraction of barred galaxies as MRK galaxies. This is a result of a correlation between properties of galaxies in pairs. However, because of magnitude limitation of Markarian survey, MRK galaxies are usually the brightest members of pairs.

The main parameters describing interactions are $\mathrm{d} V, D \mathrm{p}$, and $P$. Without considering morphologies, there is a 0.7 dex increase of SSFRs from larger $\mathrm{d} V$ to smaller ones. However the variance of SSFRs because of morphologies is much larger (more than $2.5 \mathrm{dex}$ ). In Ellison et al. (2010), it was shown that pairs in denser environments have larger $\mathrm{d} V$. Because of morphology-density relation, $\mathrm{d} V$ is also biased by morphologies: early-type galaxies have about 3 times more $\mathrm{d} V$ than irregulars, so most of the SSFR vs. $\mathrm{d} V$ dependence is because of morphology-SSFR dependence and does not reflect pure interaction. Therefore, it is essential to take into account morphologies of galaxies when discussing their SSFRs and interactions. Grouping by morphologies weakens SSFR vs. $\mathrm{d} V$ relation, but there still remains some variance, maximal for early spirals $(0.4-0.5$ dex $)$. This result is in agreement with modeling, e.g. Di Matteo et al. (2008) showing that strong starbursts during interactions are rare and that typical enhancement of SFR is less than 5 times. Figure 1 shows the dependence of SSFR on $D$ p of a pair in AGN-removed 


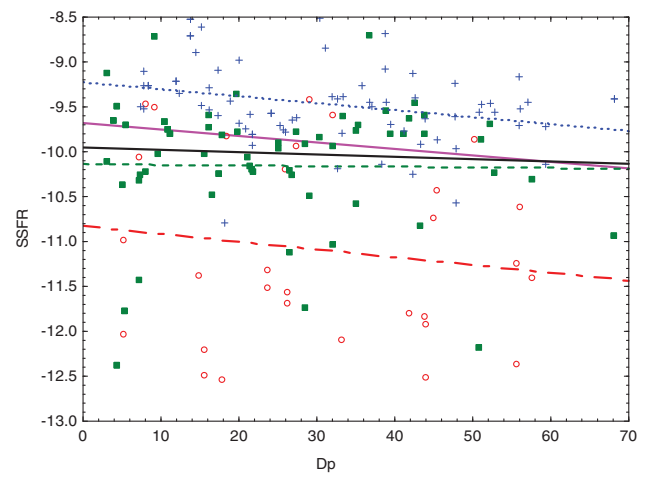

Figure 1. SSFR vs. $D$ p for subsamples of early-types (red blank circles, dashed-dotted line), early spirals (green filled squares, dashed line), and late spirals and irregulars (blue crosses, dotted line). Two best-fit lines for all galaxies (black bottom solid line) and AGN-removed sample (purple upper solid line) are drawn.

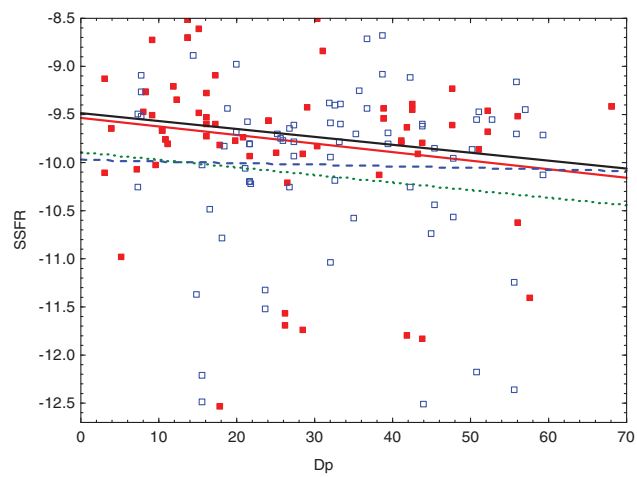

Figure 2. SSFR vs. $D p$ for major interactions (red filled squares, solid line best fit), minor interactions (blue blank squares, dashed line), brightest components of major interactions (black upper solid line), and brightest components of minor interactions (green dotted line).

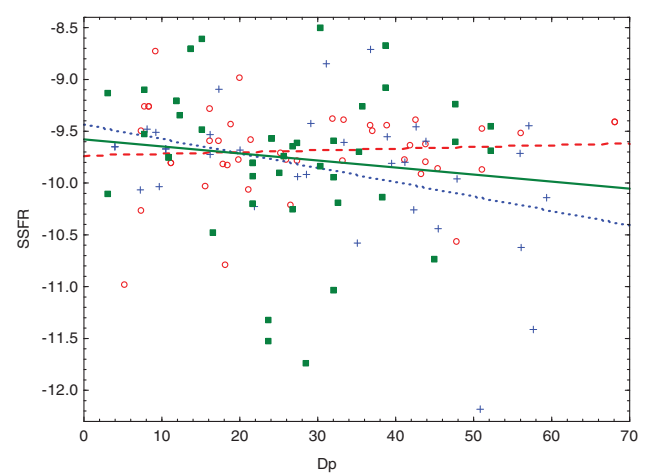

Figure 3. SSFR vs. $D$ p for galaxies with relatively earlier type neighbors (red blank circles, dashed line), same type neighbors (green filled squares, solid line), and relatively later type neighbors (blue crosses, dotted line).

sample. In our sample $D \mathrm{p}$ is not biased by morphologies compared to $\mathrm{d} V$. After grouping by morphologies we have about 0.5 dex increase of SSFRs in closer pairs.

We studied the impact of the luminosity ratio of pair members on SSFR increase by considering major $\left(\log \left(L_{\text {bright }} / L_{\text {faint }}\right) \leqslant 0.6\right)$ and minor (the rest) pairs. The major interactions are more effective in triggering SF than minor ones (see Fig. 2), there is a 0.5 dex increase of SF in major interactions, while there is no trend among minor interactions. This results are in agreement with both observational and modeling data (Lambas et al. 2003, 2012, Cox et al. 2008 ) suggesting that the merger mass ratio is an important parameter defining the effectiveness of the tidal forces.

The impact of morphology of neighbor galaxy on SSFR is shown in Fig. 3. Earliertype neighbors do not increase of SSFR, while the same- and later-type neighbors increase SSFR. The extra SSFR is maximal if the neighbor is of later morphological type (0.8 dex). Previous papers, e.g. Hwang et al. (2010), also obtained similar result, supporting the 
scenario where neighbor of later type can not only trigger gas inflow in earlier type galaxy, but also be an additional source of gas fuel.

The fraction of AGN galaxies in less separated pairs is larger than that in more separated pairs. Fraction of AGNs increases by about 7 times when going from unperturbed pairs $(P=0)$ to mergers $(P=3)$. This result indicates that while both AGN and SF can be triggered by interactions, AGNs "prefer" stronger interactions. Different timings of starbursts and AGN events can explain this result (Ellison et al. 2008, Wild et al. 2010, Hopkins 2012). The fraction of AGNs in major interactions is about 4 times larger than that in minor ones.

We studied BPT-BPT correlations between pair members. There is a tendency to have an increased probability of a neighbor with the same BPT type. Especially that is noticeable regarding passive galaxies and AGNs: passive galaxies are more likely to be found near another passive galaxy, AGNs are more probable to be near another AGN. We explain these correlations between BPT types as a result of two main factors: first, the correlation between morphologies of galaxies, and, second, the increased likelihood of pair members to have nuclear activity of same types when interacting strongly.

\section{Acknowledgement}

A. R. P. and A. A. H. acknowledge the hospitality of the Institut d'Astrophysique de Paris (France) during their stay as visiting scientists supported by the Collaborative Bilateral Research Project of the State Committee of Science (SCS) of the Republic of Armenia and the French Centre National de la Recherché Scientifique (CNRS). This work was made possible in part by a research grant from the Armenian National Science and Education Fund (ANSEF) based in New York, USA.

\section{References}

Cox, T. J., Jonsson, P., Somerville, R. S., et al. 2008, MNRAS, 384, 386

Di Matteo, P., Bournaud, F., Martig, M., et al. 2008, A\&A, 492, 31

Ellison, S. L., Patton, D. R., Simard, L., \& McConnachie, A. W. 2008, AJ, 135, 1877

Ellison, S. L., Patton, D. R., Simard, L., et al. 2010, MNRAS, 407, 1514

Ellison, S. L., Patton, D. R., Mendel, J. T., \& Scudder, J. M. 2011, MNRAS, 418, 2043

Ellison, S. L., Mendel, J. T., Scudder, J. M., et al. 2013, MNRAS, 430, 3128

Hopkins, P. F. 2012, MNRAS, 420, L8

Hopkins, P. F., Cox, T. J., Hernquist, L., et al. 2013, MNRAS, 430, 1901

Hwang, H. S., Elbaz, D., Lee, J. C., et al. 2010, A\&A, 522, A33

Lambas, D. G., Tissera, P. B., Alonso, M. S., \& Coldwell, G. 2003, MNRAS, 346, 1189

Lambas, D. G., Alonso, S., Mesa, V., \& O’Mill, A. L. 2012, A\&A, 539, A45

Larson, R. B., \& Tinsley, B. M. 1978, ApJ, 219, 46

Li, C., Kauffmann, G., Heckman T. M., et al. 2008, MNRAS, 385, 1903

Liu, X., Shen, Y., \& Strauss, M. A. 2012, ApJ, 745, 94

Markarian, B. E., Lipovetsky, V. A., Stepanian, J. A., et al. 1989, Comm. SAO, 62, 5

Mihos, J. C., Hernquist, L. 1996, ApJ, 464, 641

Nazaryan, T. A., Petrosian, A. R., \& McLean, B. J. 2012, Astrophys., 55, 448

Nazaryan, T. A., Petrosian, A. R., Hakobyan, A. A., et al. 2014, Astrophys., 57, 14

Nazaryan, T. A. 2014, Astrophys., 57, 50

Petrosian, A., McLean, B., Allen, R. J., \& MacKenty, J. W. 2007, ApJS, 170, 33

Scudder, J. M., Ellison, S. L., \& Mendel, J. T. 2012, MNRAS, 423, 2690

Sol Alonso, M., Lambas, D. G., Tissera, P., \& Coldwell, G. 2006, MNRAS, 367, 1029

Wild, V., Heckman, T., \& Charlot, S. 2010, MNRAS, 405, 933 